\title{
Vibration of Visco-elastic Orthotropic Parallelogram Plate with Linear Thickness Variation in Both Directions
}

\author{
Arun Kumar Gupta \\ M. S. College, Saharanpur, U.P., India \\ Amit Kumar \\ Shivaji College, University of Delhi, Delhi, India \\ Harvinder Kaur \\ Govt.College, Ambala, Haryana, India
}

(Received 29 January 201; provisionally accepted 6 September 2010; officially accepted 27 September 2010)

\begin{abstract}
A simple model presented here for vibration of a visco-elastic, orthotropic parallelogram plate with linear thickness variation in both directions. Using the separation of variables method, the governing differential equation has been solved for the vibration of a visco-elastic, orthotropic parallelogram plate having clamped boundary conditions on all four of the edges. An approximate but convenient frequency equation is derived by using the Rayleigh-Ritz technique with a two-term deflection function. The time period and deflection function at different points for the first two modes of vibration are calculated for various values of taper constants, aspect ratio and skew angle.
\end{abstract}

\section{INTRODUCTION}

Structures of plates have wide applications in ships, aircrafts, bridges, and so on. A thorough dynamic study of their behavior and characteristics is essential to assess and use the full potentials of plates. In the aeronautical field, analysis of plates with variable thickness has been of great interest due to their utility in aircraft wings. Srinivasan et al have discussed the analysis of skew orthotropic point supported plates. ${ }^{1}$ Gutierrez et al solved the higher frequencies of transverse vibration of rectangular plates elastically restrained against rotation at the edges and with a central free hole. ${ }^{2}$ Transverse vibration of triangular plates using characteristic orthogonal polynomials in two variables have discussed by Singh et al. ${ }^{3}$ Nair et al have solved the problem of vibration of skew plates. ${ }^{4}$ Reddy et al discuss the free vibration of skew laminates. ${ }^{5}$ Vibration of skew plates using orthogonal polynomials has been discussed by Singh et al. ${ }^{6}$ Gupta et al have solved vibration problems of visco-elastic rectangular plate with linearly thickness variations in both directions. ${ }^{7}$ Transverse vibration of skew plates with variable thickness is discussed by Singh et al. ${ }^{8}$

The vibration of clamped visco-elastic rectangular plates with parabolic thickness variations has been discussed by Gupta et al. ${ }^{9}$ Free transverse vibration of orthotropic viscoelastic rectangular plates with continuously varying thickness and density have been discussed by Gupta et al. ${ }^{10}$ Gupta et al discuss the thermal effect on vibration of a clamped viscoelastic rectangular plate with parabolic thickness variation in both directions. ${ }^{11}$ Kumar has solved natural frequencies of rectangular orthotropic plates with a pair of simply supported parallel edges. ${ }^{12}$ Free vibration analysis of functionally graded plates using the element-free kp-Ritz Method has been discussed by Zhau et al. ${ }^{13}$ Thermoelasticity has been discussed by
Parkus ${ }^{14}$ and Nowacki. ${ }^{15}$ Vibration of clamped visco-elastic rectangular plates with bi-directional, exponential thickness variations has been discussed Gupta et al. ${ }^{16}$

A survey of the literature on vibration problems of skew plates shows that the vibration of skew plates has received less attention than that given to the other types, that is rectangular, circular, and elliptic plates. Recently, Gupta et al have studied the vibration of visco-elastic parallelogram plates with parabolic thickness. ${ }^{17}$ Sufficient work is available on the vibration of a rectangular plate of variable thickness, but none exists on a parallelogram plate. ${ }^{18,19}$

Modern engineering structures are based on different design types, which involve various types of anisotropic and nonhomogeneous materials in the form of their structural components. Depending upon the requirement, durability and reliability, materials are being developed so that they can be used to provide better strength and efficiency. The equipment used in air jets, communications, and in other similar technological industries take into consideration such materials, which not only reduce the weight and size but also are reliable in terms of efficiency, strength, and economy.

A simple model presented here is used to study the effect of linear thickness variation in both directions on the vibration of a visco-elastic, orthotropic parallelogram plate with clamped boundary conditions on all four of the edges. The hypothesis of small deflection and linear, orthotropic visco-elastic properties are made. Using the separation of variables method, the governing differential equation has been solved for vibration of a visco-elastic, orthotropic parallelogram plate. An approximate but convenient frequency equation is derived by using the Rayleigh-Ritz technique with a two-term deflection function. It is assumed that the visco-elasticity of the plate is of the Kelvin Type. The time period and deflection function at different points for the first two modes of vibration are calculated for 